\title{
Searching for Radiative Neutrino Mass Generation at the LHC
}

\author{
Raymond R. Volkas* \\ ARC Centre of Excellence for Particle Physics at the Terascale \\ School of Physics, The University of Melbourne \\ Victoria, 3010, Australia \\ *E-mail: raymondv@unimelb.edu.au
}

\begin{abstract}
In this talk, I describe the general characteristics of radiative neutrino mass models that can be probed at the LHC. I then cover the specific constraints on a new, explicit model of this type.

Keywords: Neutrino; radiative mass generation; effective operators; flavor-violation constraints; LHC constraints
\end{abstract}

\section{See-saw versus radiative neutrino mass generation}

Many models of radiative neutrino mass are possible, some of which have been analysed in depth, while others have been examined only briefly, with quite a number yet to be explicitly written down let alone analysed. A useful organising principle for Majorana neutrino mass models is to use standard model (SM) effective operators that violate lepton number conservation by two units $(\Delta L=2$ operators) as a base from which to construct new theories. $\underline{\underline{1}-7}$

Restricting ourselves to operators containing SM fermions and a single Higgs doublet, they occur at odd mass dimensions, with the lowest order one occurring at $d=5$. That operator is the famous Weinberg operator, which has the schematic structure $L L H H$, where $L$ is a lepton doublet and $H$ the Higgs doublet. After electroweak symmetry breaking, this operator (actually a set of operators because of the family structure) directly induces a Majorana neutrino mass for left-handed (LH) neutrinos given by the see-saw formula,

$$
m_{\nu} \sim \frac{\langle H\rangle^{2}}{M}
$$

where $M$ is the scale of the new physics that gives rise to the operator in the low-energy limit.

Underlying renormalisable theories yielding $L L H H$ are constructed by "opening up" the operator. The type-1,-2 and -3 see-saw models are the minimal, tree-level ways to open up $L L H H . \sqrt[8-19]{ }$ By starting with the effective operator, one may systematically construct all of the minimal underlying models.

Other $\Delta L=2$ effective operators have mass dimension seven or higher and feature other lepton and quark fields in their expression, except for those in the 
generalised Weinberg class $L L H H(\bar{H} H)^{n}$. 3.5.7 These additional particles have to be closed off in loops to produce a Majorana neutrino mass self-energy graph from the operator. Thus, theories based on $d \geq 7$ operators (apart from the generalised Weinberg class) necessarily produce radiative neutrino mass generation. Underlying renormalisable theories can then be systematically constructed by opening up the operators in all possible ways, subject in practice to minimality assumptions, just as the three see-saw models may be derived from the Weinberg operator.

A list of all gauge invariant $\Delta L=2$ operators at $d=5,7,9,11$, constructed from SM fermions and one Higgs doublet, was produced by Babu and Leung (BL). 1 The ninth operator in their list, $O_{9}=L L L e^{c} L e^{c}$ (which has $d=9$ ), is the basis of the historically important Zee-Babu model of neutrino mass generation. ${ }^{20.21}$ Two exotic scalars are introduced: an isosinglet, singly-charged state $h$ coupling to $L L$ and a doubly-charged, isosinglet $k$ coupling to $e^{c} e^{c}$. Both carry two units of lepton number. The cubic term $h h k$ combines with those interactions to induce $\Delta L=2$ and produce Majorana neutrino mass at 2-loop order. ATLAS has searched for $k$ through the same-sign dilepton channels $e e, e \mu$ and $\mu \mu$, deriving a lower bound of about $320 \mathrm{GeV}$ on the mass 22 (see also 23 ).

It is nice that the $\Delta L=2$ operator perspective places the radiative neutrino mass models at one end of a systematic list of Majorana mass models, bookended by the tree-level see-saw models at $d=5$. From the phenomenological viewpoint, radiative models are interesting because the mass scale of the new physics is lower than the favoured very high scale for see-saw models, and has more chance of encroaching into the LHC regime.

It is very much worth noting that upper bounds on the see-saw scales can be derived from naturalness considerations, because the new particles and interactions will destabilise the electroweak scale if the new physics occurs at too high a scale. The type- 1 upper bound was first computed by Vissani 24 to be $3 \times 10^{7} \mathrm{GeV}$, if the Higgs self-energy graph from neutrino Yukawa interactions is not to produce a correction to the $\mu^{2}$ parameter in the Higgs potential greater than $(1 \mathrm{TeV})^{2}$. This one-family result has recently been generalised to the realistic three-family case,, 25 producing the bounds

$$
M_{N_{1}} \lesssim 4 \times 10^{7} \mathrm{GeV}, \quad M_{N_{2}} \lesssim 7 \times 10^{7} \mathrm{GeV}, \quad M_{N_{3}} \lesssim 3 \times 10^{7} \mathrm{GeV}\left(\frac{0.05 \mathrm{eV}}{m_{\min }}\right),
$$

where $N_{1,2,3}$ are the three heavy neutral lepton mass eigenstates with $M_{N_{1}} \leq M_{N_{2}} \leq$ $M_{N_{3}}$, and $m_{\min }$ is the minimum light neutrino mass. It is interesting that these bounds imply that standard thermal, hierarchical leptogenesis 26 , which requires sufficiently massive heavy neutral leptons, must involve some level of fine-tuning for the minimal type- 1 see-saw model. This is true for all three cases: $N_{1}, N_{2}$ and $N_{3}$ leptogenesis. Naturalness, if successful leptogenesis is desired, may be restored by extending the theory. An obvious example is the supersymmetric extension, and another is to add extra Higgs doublets, to divorce the leptogenesis parameter space from neutrino mass generation (one version of this is discussed in Ref. $\frac{27}{}$ ). 


\section{Opening up $d=7$ operators}

Let us examine the $\Delta L=2, d=7$ operators from the BL list as the next most complicated cases after the Weinberg operator. The flavour contents are

$$
O_{2}=L L L e^{c} H, \quad O_{3}=L L Q d^{c} H, \quad O_{4}=L L \bar{Q} \bar{u}^{c} H, \quad O_{8}=L \bar{e}^{c} \bar{u}^{c} d^{c} H,
$$

where the BL numbering scheme has been used, with $Q$ the quark doublet, $d$ the $\mathrm{RH}$ down quark and $u$ the $\mathrm{RH}$ up quark. Flavour structures $O_{3,4}$ each yield two independent operators once weak-isospin index contraction possibilities are taken into account. Operator $O_{3}$ is the basis of the pioneering 1-loop Zee model, ${ }^{28}$ while models constructed from $O_{3}$ and $O_{8}$ have been analysed in depth by Babu and Julio. ${ }^{29.30}$ In addition, $d=7$ contains the Weinberg-operator generalisation $O_{1}^{\prime}=$ $L L \bar{H} H H H .3 .7$

We adopt the minimality assumption that the underlying renormalisable theories obtained from opening up these operators contain only exotic heavy scalars and vector-like fermions. Table 1 lists the quantum numbers under $\mathrm{SU}(3) \times \mathrm{SU}(2) \times \mathrm{U}(1)$ for scalar-only and scalar-fermion extensions. 31 The example in boldface will be analysed in detail below. The diagram topologies are given in Fig. 11

Table 1. Quantum numbers of exotic scalars and fermions in underlying theories for the $d=7$ operators.

\begin{tabular}{cccc}
\hline Scalar & Scalar & Dirac fermion & Operator \\
\hline$(1,2,1 / 2)$ & $(1,1,1)$ & & $O_{2,3,4}$ \\
$(3,2,1 / 6)$ & $(3,1,-1 / 3)$ & & $O_{3,8}$ \\
$(3,2,1 / 6)$ & $(3,3,-1 / 3)$ & & $O_{3}$ \\
$(1,1,1)$ & & $(1,2,-3 / 2)$ & $O_{2}$ \\
$(1,1,1)$ & & $(3,2,-5 / 6)$ & $O_{3}$ \\
$(1,1,1)$ & & $(3,1,2 / 3)$ & $O_{3}$ \\
$(3,2,1 / 6)$ & & $(3,2,-5 / 6)$ & $O_{3}$ \\
$(\mathbf{3 , 1}, \mathbf{- 1 / 3})$ & & $(\mathbf{3 , 2},-\mathbf{5} / \mathbf{6})$ & $\mathbf{O}_{3,8}$ \\
$(3,3,-1 / 3)$ & & $(3,2,-5 / 6)$ & $O_{3}$ \\
$(3,2,1 / 6)$ & & $(3,3,2 / 3)$ & $O_{3}$ \\
$(1,1,1)$ & & $(3,2,7 / 6)$ & $O_{4}$ \\
$(1,1,1)$ & & $(3,1,-1 / 3)$ & $O_{4}$ \\
$(3,2,1 / 6)$ & & $(3,2,7 / 6)$ & $O_{8}$ \\
$(3,2,1 / 6)$ & & $(1,2,-1 / 2)$ & $O_{8}$ \\
$(1,4,3 / 2)$ & & $(1,3,-1)$ & $O_{1}^{\prime}$ \\
\hline
\end{tabular}

It is clear that searches for radiative neutrino mass models are included in general searches for exotic scalars and fermions. Each specific model has a particular scalar-scalar or scalar-fermion pair. In addition, the decay branching ratios for the exotics are typically quite constrained by the requirement to reproduce the correct neutrino masses and mixing angles. This additional information must be used when interpreting the experimental constraints, and it constitutes a "smoking gun" for exotics that are responsible for radiative neutrino mass generation. 


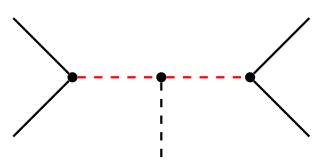

(a)

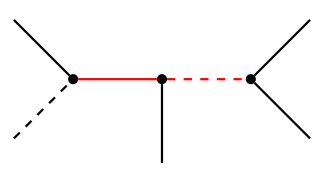

(b)

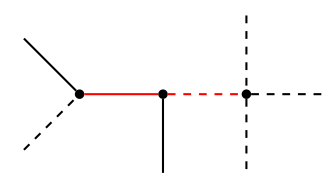

(c)

Fig. 1. Diagram topologies for opening up $d=7$ operators. (a) Scalar-only. (b) Scalar plus fermion for $O_{2,3,4,8}$. (c) Scalar plus fermion for $O_{1}^{\prime}$.

\section{Collider constraints on a new radiative model}

We now look at the boldfaced case in Table 131 Our theory will produce $O_{3}$, with a subdominant $O_{8}$. The exotic scalar $\phi$ and exotic fermion $\chi$ have the quantum numbers

$$
\phi \sim(\overline{3}, 1,1 / 3), \quad \chi \sim(3,2,-5 / 6)
$$

The Lagrangian is

$$
\begin{aligned}
-\mathcal{L} & =m_{\phi}^{2} \phi^{\dagger} \phi+m_{\chi} \bar{\chi} \chi+\left(Y_{i j}^{\bar{e} \bar{u} \phi} \bar{e}_{i} \bar{u}_{j} \phi^{\dagger}+h . c .\right) \\
& +\left(Y_{i j}^{L Q \phi} L_{i} Q_{j} \phi+Y_{i}^{L \bar{\chi} \phi} L_{i} \bar{\chi} \phi^{\dagger}+Y_{i}^{\bar{d} \chi H} \bar{d}_{i} \chi H+\text { h.c. }\right)
\end{aligned}
$$

where we use two-component notation for the fermions and $i, j$ are family indices. The Yukawa coupling constants $Y^{\bar{e} \bar{u} \phi}$ play no role in neutrino mass generation and are thus set to zero for simplicity. We also impose baryon-number conservation to forbid the $Q Q \phi^{\dagger}$ and $\bar{d} \bar{u} \phi$ interactions permitted by the gauge symmetry.

The diagram responsible for neutrino mass generation is given in Fig. 2, It is proportional to the down-type quark masses. Barring unaesthetic hierarchies in the coupling constants, the terms proportional to the $b$-quark mass will dominate, which is what we will assume is the case. Associated with that, for simplicity we switch off the mixing of $\chi$ with first- and second-generation quarks.

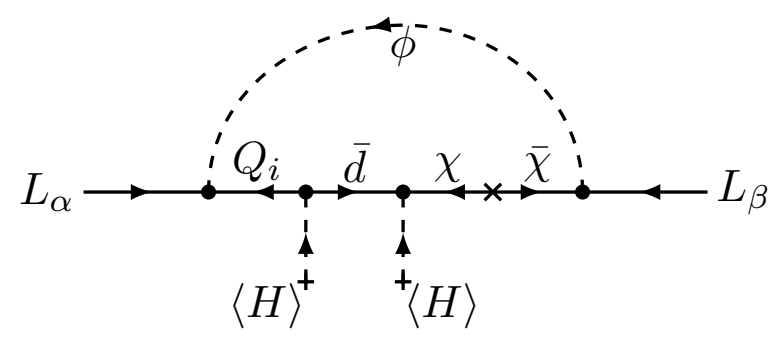

Fig. 2. Feynman diagram responsible for 1-loop generation of neutrino masses and mixings.

The neutrino mass matrix is then

$$
\left(m_{\nu}\right)_{i j}=\frac{3}{16 \pi^{2}}\left(Y_{i 3}^{L Q \phi} Y_{j}^{L \bar{\chi} \phi}+(i \leftrightarrow j)\right) m_{b B} \frac{m_{b} m_{B}}{m_{\phi}^{2}-m_{B}^{2}} \ln \frac{m_{B}^{2}}{m_{\phi}^{2}} .
$$




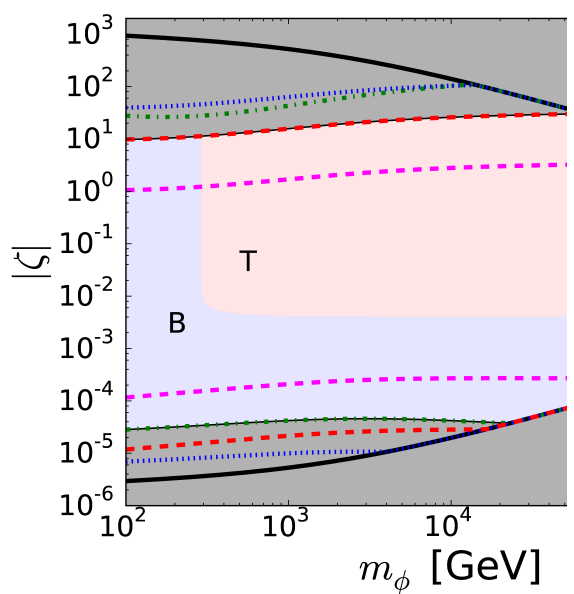

(a)

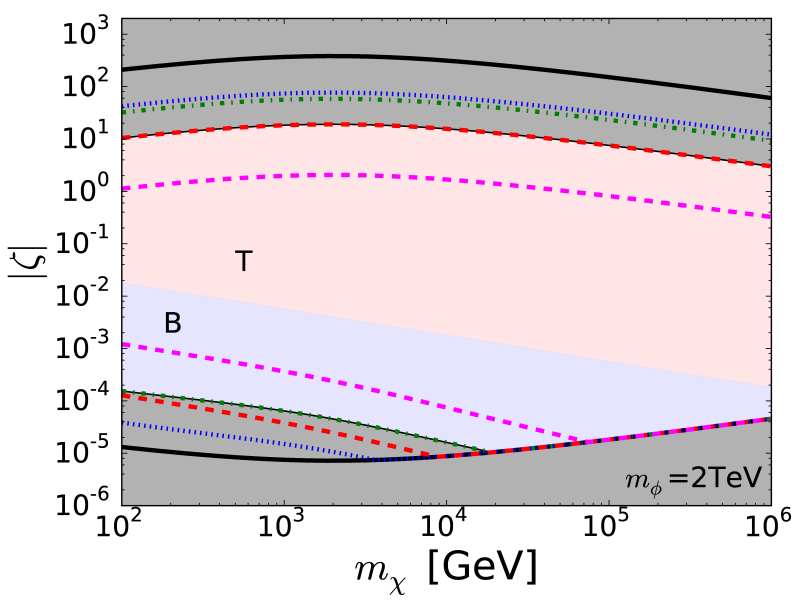

(b)

Fig. 3. Lepton flavour violation bounds. (a) Allowed region in the $|\zeta|, m_{\phi}$ plane for $m_{\chi}=2 \mathrm{TeV}$. (b) Allowed region in the $|\zeta|, m_{\chi}$ plane for $m_{\phi}=2 \mathrm{TeV}$.

The bottom quark mixes with the isospin $+1 / 2$ component of $\chi$ to form two mass eigenstates, $b$ and $B$, with masses $m_{b}$ and $m_{B}$ respectively. The parameter $m_{b B}=$ $Y_{3}^{\bar{d} \chi H} v / \sqrt{2}$, where $\left\langle H^{0}\right\rangle=v / \sqrt{2}$. At this level of approximation, there are two massive neutrinos and a massless one. In reality, the lightest neutrino will pick up a small mass, but its value is unimportant and will be neglected. CP violating phases will also be set to zero for simplicity.

The rank-2 mass matrix may be expressed as the symmetrised outer product of two vectors $a_{ \pm}$,

$$
m_{\nu}=a_{+} a_{-}^{T}+a_{-} a_{+}^{T},
$$

where

$$
a_{ \pm}^{\mathrm{NO}}=\frac{\zeta^{ \pm 1}}{\sqrt{2}}\left(\sqrt{m_{2}} u_{2}^{*} \pm i \sqrt{m_{3}} u_{3}^{*}\right), \quad a_{ \pm}^{\mathrm{IO}}=\frac{\zeta^{ \pm 1}}{\sqrt{2}}\left(\sqrt{m_{1}} u_{1}^{*} \pm i \sqrt{m_{2}} u_{2}^{*}\right)
$$

with NO and IO denoting normal and inverted ordering, respectively. The vectors $u_{1,2,3}$ are the columns of the PMNS matrix, and the complex number $\zeta$ is a Casas-Ibarra-like parameter, not determined by low-energy experiments. Equating 6 and 7 constrains the parameter space to the region compatible with the neutrino oscillation results.

The next set of constraints on the parameter space come from the lepton flavour violating processes $\mu \rightarrow e \gamma, \mu \rightarrow e e e$ and $\mu N \rightarrow e N . \underline{31}$ An example of the results is presented in Fig. 3. The green dash-dotted, blue dotted and red-dashed lines bound the regions corresponding to $\mathrm{BR}(\mu \rightarrow e \gamma)<5.7 \times 10^{-13}, \mathrm{BR}(\mu \rightarrow e e e)<10^{-12}$ and $\mathrm{BR}(\mu \mathrm{Au} \rightarrow e \mathrm{Au})<7 \times 10^{-13}$, respectively. The grey region is thus excluded. The magenta dashed line shows the reach of the $\mu \mathrm{Ti} \rightarrow e \mathrm{Ti} \mathrm{Mu} 2 \mathrm{E}$ and COMET experiments. The allowed region is divided into $B$ and $T$ regions in which $\operatorname{BR}(\phi \rightarrow$ $b \nu)=1$ and $\operatorname{BR}(\phi \rightarrow b \nu)<1$, respectively.

The vector-like fermion doublet $\chi$ consists of the charge $-1 / 3$ quark $B^{\prime}$ which mixes with the bottom quark, and an exotic charge $-4 / 3$ quark $Y$. CMS have searched for $B$-like particles, whose dominant decay modes are $B \rightarrow Z b$ and $B \rightarrow H b$ in our model with branching ratios that are strongly constrained by the need to fit 
the neutrino oscillation data. Taking that into account, the CMS bound is $m_{B} \gtrsim 620$ $\mathrm{GeV} .{ }^{32-36}$ No $Y$ search has been performed.

The other collider constraint comes from searches for leptoquark scalar $\phi$. It is pair-produced at the LHC from gluon-gluon fusion and $q \bar{q}$ annihilation. Since the production is through its colour charge, the rate depends only on $m_{\phi}$. For example, for $m_{\phi}=500(600) \mathrm{GeV}$, the production cross-section $\sigma(p p \rightarrow \phi \phi)$ is $82(23.5) \mathrm{fb}$.

The main decay modes are into $L t$ and $b \nu$, where $L=(e, \mu, \tau)$. In the parameter region $m_{Y, B} \gg m_{\phi}$, so that $L Y$ and $B \nu$ final states are kinematically forbidden, the partial decay rates are,

$$
\begin{aligned}
\Gamma(\phi \rightarrow L t) & =\frac{m_{\phi}}{8 \pi}\left|Y_{L 3}^{L Q \phi}\right|^{2} f\left(m_{\phi}, m_{L}, m_{t}\right), \\
\Gamma\left(\phi \rightarrow \nu_{L} b\right) & \simeq \frac{m_{\phi}}{8 \pi}\left(\left|Y_{L 3}^{L Q \phi} \cos \theta_{2}\right|^{2}+\left|Y_{L}^{L \bar{\chi} \phi} \sin \theta_{1}\right|^{2}\right) f\left(m_{\phi}, m_{L}, m_{t}\right),
\end{aligned}
$$

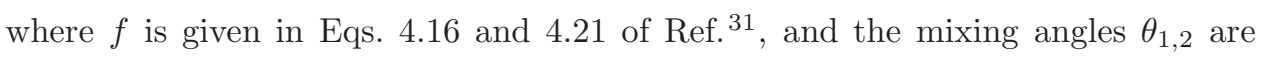
defined through

$$
\sin \theta_{1}=\frac{m_{b B} m_{\chi}}{m_{\chi}^{2}-m_{b^{\prime}}^{2}}, \quad \sin \theta_{2}=\frac{m_{b B} m_{b^{\prime}}}{m_{\chi}^{2}-m_{b^{\prime}}^{2}},
$$

where $m_{b^{\prime}}=y_{b} v / \sqrt{2}$ is the bottom quark mass in the absence of mixing with $B^{\prime}$. The decay rates depend on the same Yukawa coupling constants that also contribute to neutrino mass generation, so are quite constrained, as well as $|\zeta|$.

Recall that the allowed regions $B$ and $T$ in Fig. 3 correspond to regimes where $\mathrm{BR}(\phi \rightarrow b \nu)=1$ and $\mathrm{BR}(\phi \rightarrow b \nu)<1$, respectively. In the $B$ region, the main signature is thus $p p \rightarrow \phi \phi \rightarrow b b+$ missing $E_{T}$, where sbottom pair searches apply. The bound in this case is $m_{\phi} \gtrsim 730 \mathrm{GeV}$ at $95 \%$ C.L. $\stackrel{37.38}{ }$ The analysis for region $T$ requires recasting stop searches as well and is summarised in Fig. 4 which plots the various branching ratios as functions of $m_{\phi}$, as well as the limits from ATLAS and CMS. ${ }^{37-41}$ In numbers: The $m_{\phi}$ lower limit from CMS in the $b b+$ missing $E_{T}$ channel is in the range $520-600 \mathrm{GeV}$. From the $(e, \mu)+$ missing $E_{T}+(b-)$ jets, ATLAS sets a limit of approximately $580 \mathrm{GeV}$. Finally, the $(e, \mu)^{+}(e, \mu)^{-}+$missing $E_{T}+$ jets channel yields about a $620 \mathrm{GeV}$ limit from ATLAS data.

\section{Final remarks}

A systematic procedure for constructing radiative Majorana neutrino mass models has been developed from standard model $\Delta L=2$ effective operators containing leptons, quarks and the Higgs doublet. The LHC is a useful tool for searching for the exotic scalars and fermions that appear in these models, and specific bounds for a new model were summarised in this talk. The $13 \mathrm{TeV}$ LHC will extend the reach of past searches. Some naturalness concerns for hierarchical, thermal leptogenesis in the minimal type- 1 see-saw model were also mentioned in passing. 


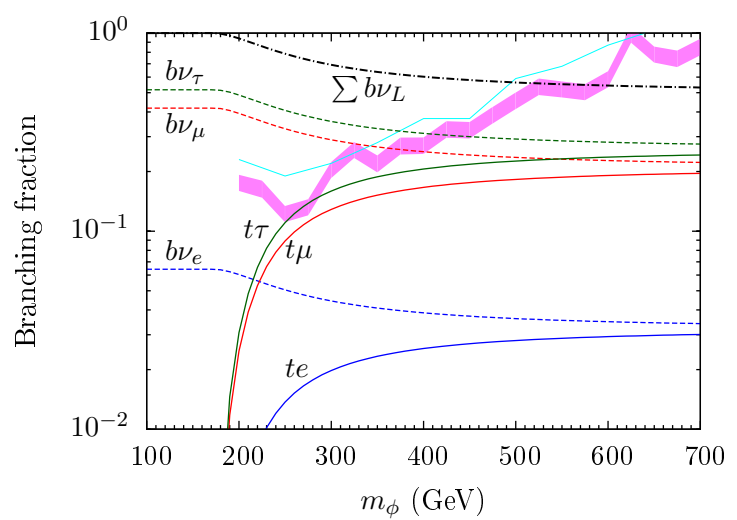

Fig. 4. Decay branching ratios of $\phi$ as a function of $m_{\phi}$ in region T. The curves with positive slope running to the top right show the ATLAS (thin line) and CMS (thick band) limits.

\section{Acknowledgments}

This work was supported in part by the Australian Research Council. I thank my coauthors P. W. Angel, Y. Cai, J. D. Clarke, R. Foot, N. L. Rodd and M. A. Schmidt. I also thank K. S. Babu, A. de Gouvêa and W. Winter for discussions over the last few years on radiative neutrino mass generation.

\section{References}

1. K. S. Babu and C. N. Leung, Classification of effective neutrino mass operators, Nucl. Phys. B619, 667 (2001).

2. A. de Gouvea and J. Jenkins, A Survey of Lepton Number Violation Via Effective Operators, Phys.Rev. D77, p. 013008 (2008).

3. F. Bonnet, D. Hernandez, T. Ota and W. Winter, Neutrino masses from higher than $\mathrm{d}=5$ effective operators, JHEP 0910, p. 076 (2009).

4. P. W. Angel, N. L. Rodd and R. R. Volkas, Origin of neutrino masses at the LHC: $\Delta L=2$ effective operators and their ultraviolet completions, Phys.Rev. D87, p. 073007 (2013).

5. F. Bonnet, M. Hirsch, T. Ota and W. Winter, Systematic study of the $\mathrm{d}=5$ Weinberg operator at one-loop order, JHEP 1207, p. 153 (2012).

6. P. W. Angel, Y. Cai, N. L. Rodd, M. A. Schmidt and R. R. Volkas, Testable twoloop radiative neutrino mass model based on an $L L Q d^{c} Q d^{c}$ effective operator, JHEP 1310, p. 118 (2013).

7. M. B. Krauss, D. Meloni, W. Porod and W. Winter, Neutrino Mass from a d=7 Effective Operator in an SU(5) SUSY-GUT Framework (January 2013).

8. P. Minkowski, $\mu \rightarrow e \gamma$ at a rate of one out of 1-billion muon decays?, Phys. Lett. B67, p. 421 (1977).

9. T. Yanagida, Horizontal gauge symmetry and masses of neutrinos, in Proceed- 
ings of the Workshop on The Unified Theory and the Baryon Number in the Universe, eds. O. Sawada and A. Sugamoto1979.

10. S. L. Glashow, The future of elementary particle physics, in Proceedings of the 1979 Cargèse Summer Institute on Quarks and Leptons, eds. M. L. vy, J.-L. Basdevant, D. Speiser, J. Weyers, R. Gastmans and M. Jacob (Plenum Press, New York, 1980).

11. M. Gell-Mann, P. Ramond and R. Slansky, Complex spinors and unified theories, in Supergravity, eds. P. van Nieuwenhuizen and D. Z. Freedman (North Holland, Amsterdam, 1979).

12. R. N. Mohapatra and G. Senjanović, Neutrino mass and spontaneous parity violation, Phys. Rev. Lett. 44, p. 912 (1980).

13. M. Magg and C. Wetterich, Neutrino mass problem and gauge hierarchy, Phys. Lett. B94, p. 61 (1980).

14. J. Schechter and J. Valle, Neutrino Masses in SU(2) x U(1) Theories, Phys.Rev. D22, p. 2227 (1980).

15. C. Wetterich, Neutrino masses and the scale of $B-L$ violation, Nucl. Phys. B187, p. 343 (1981).

16. G. Lazarides, Q. Shafi and C. Wetterich, Proton lifetime and fermion masses in an SO(10) model, Nucl. Phys. B181, p. 287 (1981).

17. R. N. Mohapatra and G. Senjanovic, Neutrino Masses and Mixings in Gauge Models with Spontaneous Parity Violation, Phys.Rev. D23, p. 165 (1981).

18. T. Cheng and L.-F. Li, Neutrino Masses, Mixings and Oscillations in SU(2) x U(1) Models of Electroweak Interactions, Phys.Rev. D22, p. 2860 (1980).

19. R. Foot, H. Lew, X. He and G. C. Joshi, SEESAW NEUTRINO MASSES INDUCED BY A TRIPLET OF LEPTONS, Z.Phys. C44, p. 441 (1989).

20. A. Zee, QUANTUM NUMBERS OF MAJORANA NEUTRINO MASSES, Nucl.Phys. B264, p. 99 (1986).

21. K. Babu, Model of 'Calculable' Majorana Neutrino Masses, Phys.Lett. B203, p. 132 (1988).

22. G. Aad et al., Search for doubly-charged Higgs bosons in like-sign dilepton final states at $\sqrt{s}=7 \mathrm{TeV}$ with the ATLAS detector, Eur.Phys.J. C72, p. 2244 (2012).

23. S. Chatrchyan et al., A search for a doubly-charged Higgs boson in $p p$ collisions at $\sqrt{s}=7 \mathrm{TeV}$, Eur.Phys.J. C72, p. 2189 (2012).

24. F. Vissani, Do experiments suggest a hierarchy problem?, Phys.Rev. D57, 7027 (1998).

25. J. D. Clarke, R. Foot and R. R. Volkas, Electroweak naturalness in three-flavour Type I see-saw and implications for leptogenesis (2015).

26. M. Fukugita and T. Yanagida, Baryogenesis Without Grand Unification, Phys.Lett. B174, p. 45 (1986).

27. H. Davoudiasl and I. M. Lewis, Right-Handed Neutrinos as the Origin of the Electroweak Scale, Phys.Rev. D90, p. 033003 (2014). 
28. A. Zee, A Theory of Lepton Number Violation, Neutrino Majorana Mass, and Oscillation, Phys. Lett. B93, p. 389 (1980).

29. K. Babu and J. Julio, Two-Loop Neutrino Mass Generation through Leptoquarks, Nucl.Phys. B841, 130 (2010).

30. K. Babu and J. Julio, Radiative Neutrino Mass Generation through Vector-like Quarks, Phys.Rev. D85, p. 073005 (December 2012).

31. Y. Cai, J. D. Clarke, M. A. Schmidt and R. R. Volkas, Testing Radiative Neutrino Mass Models at the LHC, JHEP 1502, p. 161 (2015).

32. CMS Collaboration, Search for Vector-Like b' Pair Production with Multilepton Final States in pp collisions at sqrt $(s)=8 \mathrm{TeV}$, Tech. Rep. CMS-PAS-B2G13-003 (2013).

33. CMS Collaboration, Search for pair-produced vector-like quarks of charge $-1 / 3$ in lepton+jets final state in pp collisions at $\operatorname{sqrt}(\mathrm{s})=8 \mathrm{TeV}$, Tech. Rep. CMSPAS-B2G-12-019 (2012).

34. CMS Collaboration, Search for pair-produced vector-like quarks of charge $-1 / 3$ in dilepton+jets final state in pp collisions at sqrt(s) $=8 \mathrm{TeV}$, Tech. Rep. CMS-PAS-B2G-12-021 (2013).

35. S. Chatrchyan et al., Inclusive search for a vector-like T quark with charge $\frac{2}{3}$ in pp collisions at $\sqrt{s}=8 \mathrm{TeV}$, Phys.Lett. B729, 149 (2014).

36. S. Chatrchyan et al., Search for top-quark partners with charge $5 / 3$ in the same-sign dilepton final state, Phys.Rev.Lett. 112, p. 171801 (2014).

37. G. Aad et al., Search for direct third-generation squark pair production in final states with missing transverse momentum and two $b$-jets in $\sqrt{s}=8 \mathrm{TeV} p p$ collisions with the ATLAS detector, JHEP 1310, p. 189 (2013).

38. CMS Collaboration, Search for direct production of bottom squark pairs, Tech. Rep. CMS-PAS-SUS-13-018, CERN (Geneva, 2014).

39. ATLAS Collaboration, Search for direct top squark pair production in final states with one isolated lepton, jets, and missing transverse momentum in sqrts $=8$, TeV pp collisions using $21 \mathrm{fb}^{-1}$ of ATLAS data, Tech. Rep. ATLASCONF-2013-037, CERN (Geneva, 2013).

40. S. Chatrchyan et al., Search for top-squark pair production in the single-lepton final state in pp collisions at $\sqrt{s}=8 \mathrm{TeV}$, Eur.Phys.J. C73, p. 2677 (2013).

41. G. Aad et al., Search for direct top-squark pair production in final states with two leptons in pp collisions at $\sqrt{s}=8 \mathrm{TeV}$ with the ATLAS detector (2014). 